\title{
Environment and Galaxy Host Properties of PTA and eLISA Sources
}

\author{
Eva Martínez Palafox ${ }^{1}$, Octavio Valenzuela ${ }^{1}$, Pedro Colín $^{2}$ and \\ Stefan Gottlöber ${ }^{3}$
${ }^{1}$ Instituto de Astronomía, Universidad Nacional Autónoma de México, A.P. 70-264, Ciudad Universitaria, D.F., México. email: evam@astro.unam.mx
${ }^{2}$ Instituto de Radioastronomía y Astrofísica, Universidad Nacional Autónoma de México, A.P. 72-3 (Xangari), Morelia, Michoacán 58089, México.
${ }^{3}$ Leibniz Institute for Astrophysics, An der Sternwarte 16, D-14482 Potsdam, Germany.

\begin{abstract}
We use a dynamical model for the decay of supermassive black hole binaries (BHBs) coupled with a cosmological simulation and semi-empirical approaches to the occupation of halos by galaxies and $\mathrm{BHs}$, in order to investigate the properties of galaxies hosting BHBs. We found bimodal distributions of hosts properties, with one peak corresponding to BHBs suitable to be detected by PTAs and the other to BHBs in the eLISA frequency window. We note that for some of the scenarios explored there must be a considerable population of such sources in the nearby Universe that might show detectable EM signatures, representing an important laboratory for multimessenger astrophysics.
\end{abstract}

Keywords. galaxies: kinematics and dynamics, galaxies: nuclei, black hole physics

\section{Introduction to the model and scenarios considered}

BHBs would comprise the strongest sources of gravitational waves (GW) once they reach mili-pc separations, for both pulsar timing arrays (PTAs) and space based detectors (such as eLISA). While BHBs coalescences constitute a natural outcome of the cosmological standard model and galaxy mergers, their dynamical evolution is still poorly understood and therefore their abundances at different stages (see Colpi 2014 for a recent and comprehensive review).

We developed a model to follow the BHB dynamical evolution (Martinez Palafox et al. 2014). First, we associate galaxies in dark matter halos from a cosmological simulation. This galaxies fulfil relationships such as $M_{\bullet}-\sigma$, spheroid mass-size $\left(M_{b}-R_{e}\right)$ and halostellar mass $\left(M_{\mathrm{H}}-M_{s}\right)$; reproduce the local red/blue fraction at $z=0$, and are in good agreement with the Tully-Fisher relation in the relevant stellar mass range $\left(M_{s}>\right.$ $\left.1 \times 10^{9} M_{\odot}\right)$. Then, we use two $\mathrm{BH}$ seeding prescriptions: an empirical relation between BH-total mass (Ferrarese 2002) and a conditional BH mass function per halo mass bin, that reproduces the local BH mass density (Degraf et al. 2011), F and O prescriptions, respectively hereafter.

Then BHBs are considered to evolve via four stages, identified by the separation between BHs and the main interactions with the surrounding material. The BHB evolution starts at its last major merger (stage 1). Then waits one dynamical time-scale, acounting for the decay of the galaxie's baryonic components within the remnant halo (stage 2). Now, we integrate the position of the secondary $\mathrm{BH}$, while dynamical friction takes it to the centre of the newly formed galaxy (stage 3). And finally, there are two options in stage 4: BHB-gaseous disk interaction (3-disk model, Hayasaki 2009), and BHB-stellar 


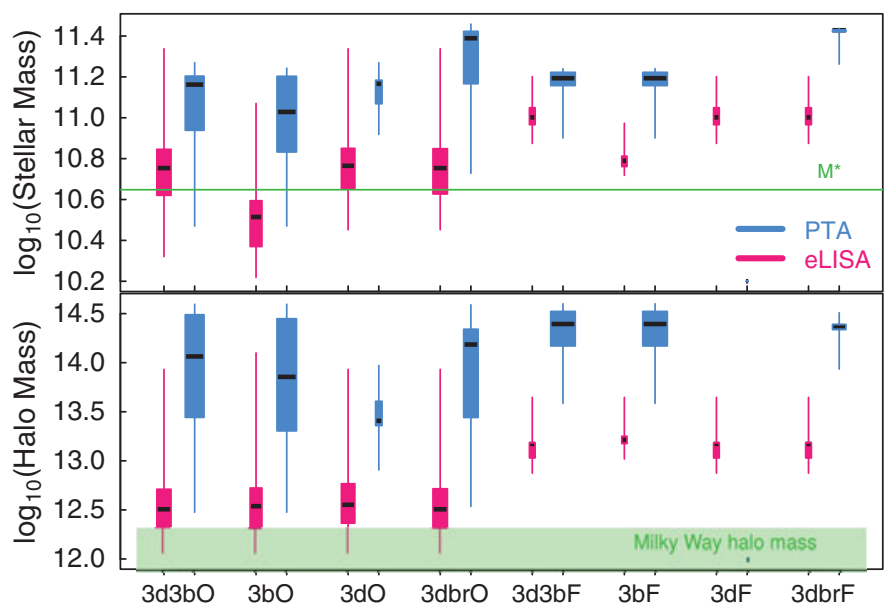

Figure 1. Halo (bottom panel) and stellar (upper panel) mass distributions represented by box and whiskers plots. The 8 scenarios split in galaxies hosting PTA (blue) and eLISA (pink) candidates. In both panels, the box show the interquartile range (IQR) where the central $50 \%$ of the data falls. Each whisker or solid line represents the lower and upper 25\%. Black lines show the median value. The box width is proportional to the square-root of the number of binaries in each distribution.

background interaction plus GW emission (3-body scattering model, Sesana \& Khan 2015). We explore this two posibilities in four different scenarios. 3d3b: If the binary dwells in a blue galaxy, it only interacts with a circumbinary gaseous disk; whilst binaries within red galaxies evolve through the interaction with the stellar background. 3b: Only binary-stellar background interaction in red and blue galaxies. 3d: Only binary-gaseous disk interaction in blue galaxies, no contribution from red galaxies. 3dbr: Only binarygaseous disk interaction in blue and red galaxies. We trace the BHBs evolution until the GW driven decay regime is reached (mili-pc separations).

\section{Summary and conclusions}

The BHBs populations, regardless of the scenario, have some common characteristics. For instance, the maximum number density of binaries occur around $z \sim 0.3$ with a typical value of $n_{\text {bin }} \sim 3 \times 10^{-5} \mathrm{Mpc}^{-3}$ (within $0.2 \mathrm{dex}$ ). The local number density of the BHs more massive than $10^{6} M_{\odot}$ is $n_{\bullet} \simeq 1.7 \times 10^{-2} \mathrm{Mpc}^{-3}$ (Shankhar et al. 2004). However, if we split the binaries in those with masses above/below $10^{8} M_{\odot}$ we create populations of binaries in the PTA/eLISA frequency window, with well defined host characteristics (Figure 1) that depend on the efficiency of the mechanism driving the orbital decay and $\mathrm{BH}$-seeding prescription.

\section{References}

Colpi, M. 2014, SSRv, 183, 189

Hayasaki, K. 2009, PASJ, 61, 65

Martínez Palafox, E., Valenzuela, O., Colín P., \& Gottlöber S. 2014; arXiv:1410.3563

Sesana, A. \& Khan, F. M. 2015, MNRAS, 454, L66

Shen, S., Mo, H. J., White, S. D. M., et al. 2003, MNRAS, 343, 978 